\title{
Can the neutron-capture cross sections be measured with Coulomb dissociation?
}

\author{
Á. Horváth ${ }^{1, a}$, K. Ieki ${ }^{2}$, Á. Kiss ${ }^{1}$, A. Galonsky ${ }^{3,4}$, M. Thoennessen ${ }^{3,4}$, T. Baumann ${ }^{3}$, D. Bazin ${ }^{3}$, C.A. Bertulani ${ }^{5}$, \\ C. Bordeanu ${ }^{6}$, N. Carlin ${ }^{7}$, M. Csanád ${ }^{1}$, F. Deák ${ }^{1}$, P. DeYoung ${ }^{3,8}$, N. Frank ${ }^{3,4}$, T. Fukuchi ${ }^{2}$, Zs. Fülöp ${ }^{9}$, A. Gade ${ }^{3}$, \\ D. Galaviz ${ }^{3}$, C. Hoffman ${ }^{3,10}$, R. Izsák ${ }^{1}$, W.A. Peters ${ }^{3,4}$, H. Schelin ${ }^{11}$, A. Schiller ${ }^{3}$, R. Sugo ${ }^{2}$, Z. Seres ${ }^{12}$, and \\ G.I. Veres ${ }^{1}$ \\ 1 Department of Atomic Physics, Eötvös Loránd University, 1117 Budapest Pázmány Péter sétány 1/A, Hungary \\ 2 Department of Physics, Rikkyo University, 3 Nishi-Ikebukuro, Toshima, Tokyo 171, Japan \\ 3 National Superconducting Cyclotron Laboratory, Michigan State University, East Lansing, MI 48824, USA \\ 4 Department of Physics and Astronomy, Michigan State University, East Lansing, MI 48824, USA \\ 5 Department of Physics, University of Arizona, 1118E 4th Street, Tucson, AZ 85721, USA \\ 6 Department of Physics, University of Washington, Seattle, WA 98195, USA \\ 7 Instituto de Física, Universidade de São Paulo, Caixa Postal 66318, 05315-970, São Paulo, Brazil \\ 8 Department of Physics and Engineering, Hope College, Holland, MI 49423-9000, USA \\ 9 ATOMKI Institute of Nuclear Research, P.O.B. 51 H-4001, Debrecen, Hungary \\ 10 Department of Physics, Florida State University, Tallahassee, FL 32306, USA \\ 11 Centro Federal de Educação Tecnológica do Paraná, Avenue Sete de Setembro 3165, 80 230-901 Curitiba, Paraná, Brazil \\ 12 KFKI Research Institute for Particle and Nuclear Physics, P.O.B. 49, Budapest, 114, Hungary
}

Received: 25 August 2005 /

Published online: 28 March 2006 - C Società Italiana di Fisica / Springer-Verlag 2006

\begin{abstract}
In this paper we present first results from a ${ }^{8} \mathrm{Li}$ electromagnetic neutron-breakup experiment. Specific reactions studied were $\mathrm{Pb}\left({ }^{8} \mathrm{Li},{ }^{7} \mathrm{Li}+n\right) \mathrm{Pb}$ and $\mathrm{C}\left({ }^{8} \mathrm{Li},{ }^{7} \mathrm{Li}+n\right) \mathrm{C}$ at $41 \mathrm{MeV} /$ nucleon beam energy. This is an effort to compare the results of a Coulomb dissociation experiment with the well determined $(n, \gamma)$ reaction cross sections at astrophysical energies. The angular dependence of the cross section above 7 degree, which is the grazing angle of ${ }^{8} \mathrm{Li}-\mathrm{Pb}$ system, is similar in shape for lead and carbon and approximately proportional to $A^{2 / 3}$ in magnitude indicating that the nuclear dissociation is the main component in this region. At very forward angles the angular distributions differ significantly and the electromagnetic dissociation dominates for the lead, although the nuclear contribution is not negligible.
\end{abstract}

PACS. 25.40.Lw Radiative capture - 25.70.De Coulomb excitation

\section{Introduction}

Many astrophysical nuclear processes, such as the rprocess, involve neutron capture by short-lived nuclei. Direct measurement of these reactions are not available because one cannot make a target of these nuclei. However, there are other methods of obtaining information of the cross sections. One example is the asymptotic normalization coefficient (ANC) method [1]. Another approach is Coulomb breakup where the inverse reaction is investigated. This inverse method has been applied already in several experiments and generally good agreements with theoretical estimates are found for dissociation cross sections [2].

However, there have not been experimental investigations of the validity of the assumptions associated with

\footnotetext{
a e-mail: akos@ludens.elte.hu
}

extracting information from the inverse reaction. For example, it is known that the excited states of the resulting nucleus following neutron capture plays an important role [3]. The radioactive beam contains only ground state nuclei. Therefore, when the inverse method is applied, the contribution of capture to an excited state should be taken into account theoretically.

Another concern relates to the calculation of the neutron capture cross section from the experimental results of the dissociation. The neutron breakup can occur from either nuclear or electromagnetic interaction. The contribution of the nuclear breakup has to be measured and removed from the total yield. The proper way to do this needs verification.

This paper will present first experimental results to check the validity of the inverse method. The reaction investigated was the neutron capture of ${ }^{7} \mathrm{Li}$. This nucleus 
is stable, and the neutron capture has been measured directly in several experiments $[3,4]$ including bombardment by thermal and fast neutrons. These results showed that the capture obey a $1 / v$ law below $254 \mathrm{keV}$ where a resonance peak is located.

As the $(n, \gamma)$ reaction for ${ }^{7} \mathrm{Li}$ is well known, the inverse process can be used to provide better understanding of the Coulomb dissociation process. This is the first detailed study of a Coulomb dissociation reaction (energy dependence and angular distributions) where the associated, well-established $(n, \gamma)$ reaction is known.

\section{The method of Coulomb dissociation and its experimental difficulties}

The dissociation of nuclei passing through the strong electric field of a heavy nucleus can be described as an absorption of a virtual photon $(\gamma)$ from the target field, followed by emission of a neutron $[5]$. The $(\gamma, n)$ reaction can be then related to the inverse $(n, \gamma)$ reaction of astrophysical interest via detailed balance. The $(\gamma, n)$ cross section is much larger than the corresponding $(n, \gamma)$ cross section at astrophysical energies because of the difference in wave numbers between $\gamma$ and neutron. As the electromagnetic interaction is well known, this simple, powerful model is often used.

When applying the method, one will face several questions that can result in systematic errors. One must consider that the capture by a ground state nucleus can lead to a ground state or excited states [3]. Another question concerns the excited states of the capturing nuclei. This will give difficulties if it occurs in the dissociation process, since the dissociation into the ground state should be measured. But for those captures when the contributions from excited states are small, they can be theoretically corrected. A detailed experimental investigation can show the importance of this circumstance and would provide a way to correct for it.

The most important systematic ambiguity is the nuclear contribution in the breakup. Since the inverse of the neutron capture is only electromagnetic dissociation, the contribution of the nuclear breakup should be well understood. The general way to estimate and subtract the contribution relies on an empirical method. The most used depends on $A^{1 / 3}$ systematics. In this model, one can assume that the neutron removal can occur only at the circumference of the target viewed from the beam direction. The angular dependence of the two types of dissociation is well known. The nuclear dissociation can occur at larger scattering angles compared to the Coulomb dissociation that is limited to angles below the grazing angle. Therefore, the angular distribution of the breakup on several targets is one way to determine the correction factors for a particular target for the electromagnetic dissociation [6].

Another aspect is the calculation of the virtual photon numbers. This is theoretically well established but depends on the closest approach of the colliding nuclei. With the precise measurement of the scattering angle, it is possible to select different impact parameters of the reaction.
As the model of Coulomb dissociation is based on firstorder perturbation theory, some questions still arise due to higher-order effects (i.e., dynamical effects and relativistic effects) [7]. The neutron capture generally is dominated by an $E 1$ transition, but in the breakup there are a number of E2 virtual photons available. Experimental results of longitudinal momentum distribution of the fragment showed that the $E 1-E 2$ process can interfere [8].

These questions have never been verified experimentally and left room for systematical errors. Although these points were discussed many times for the ${ }^{8} \mathrm{~B}$ breakup reaction, which is a very important reaction in the solar neutrino problem, and several efforts have been made to clarify nuclear or E2 contributions [9], no attempt have been made to study Coulomb dissociation reaction systematics in detail.

\section{The experimental procedure}

The experiment was performed at the Coupled Cyclotron Facility of the National Superconducting Cyclotron Laboratory at Michigan State University. A ${ }^{8} \mathrm{Li}$ beam of 41.2 MeV/nucleon bombarded a lead (carbon) target of $56.7(28.8) \mathrm{mg} / \mathrm{cm}^{2}$ thickness, respectively. The experimental setup is shown in fig. 1. The direction of the incoming ${ }^{8} \mathrm{Li}$ was measured with a pair of Cathode Readout Drift Chamber (CRDC) detectors [10]. A thin plastic scintillator placed in front of the target provided the start pulse for the time of flight ( $\mathrm{ToF})$. The neutrons were detected with the Modular Neutron Array (MoNA) which consists of 9 layers of 16 scintillator bars, $10 \mathrm{~cm} \times 10 \mathrm{~cm} \times$ $2 \mathrm{~m}$ each $[11,12]$. The charged particles were deflected by the Sweeper Magnet [13] and their positions were measured after the magnet with two CRDCs separated by $1 \mathrm{~m}$. Thin and thick plastic scintillators were used to identify the particles by their energy loss. The thin plastic detector also provides trigger signal for the measurement. Measurements without any target were performed to deduce contributions from reactions in the start detector.

In the Coulomb dissociation reaction, the relative energy between ${ }^{7} \mathrm{Li}$ and $n$ is determined by their relative velocities event by event. The neutron velocity vector is deduced from the position of the scintillator bar hit in MoNA, and the ToF between the target and the particular bar. Horizontal position at MoNA is calculated from the time difference between the two photomultipliers attached at the left and right ends of each bar. The relation between time difference and position was carefully examined with cosmic-ray events. The vertical and longitudinal position of the neutron was determined from the position of the bar. The ToF was calibrated with prompt $\gamma$-rays from a thick target. The velocity was deduced from ToF between the pulse of the thin plastic scintillator and the mean time of the two photomultipliers.

To deduce the velocity of the ${ }^{7} \mathrm{Li}$ particle, more elaborate efforts were necessary. As the primary purpose of the Sweeper Magnet was to sweep the charged particles off and pass the neutrons through its large vertical gap, the magnetic field is not uniform over the whole region. To 


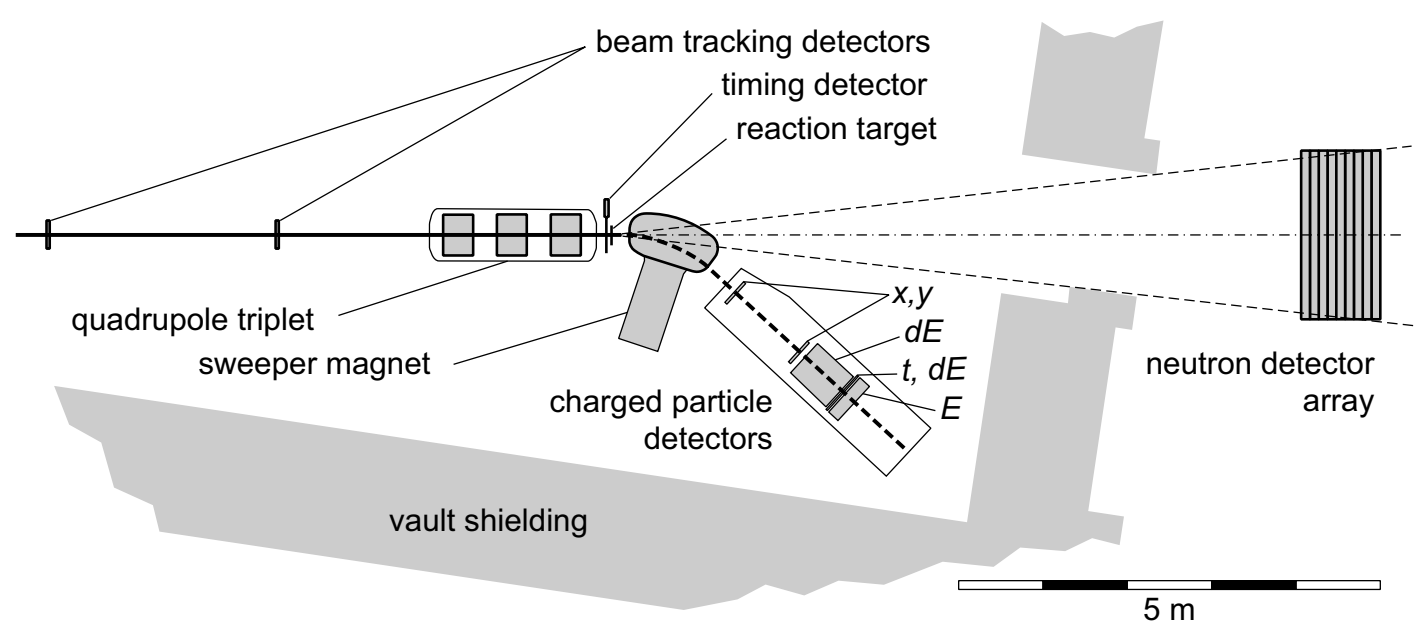

Fig. 1. Experimental setup; " $x, y$ ": CRDC detectors, " $t, d E "$ " thin plastic detector, " $E$ ": thick plastic detector.

understand the characteristics of the charged particle trajectory in the Sweeper Magnet, the program "COSY infinity" [14] was employed. From the measured magnetic field, COSY can produce a forward map, which relates position, direction and the rigidity of the particle at the target to the position and direction after the magnet. Because of the nonuniformity of the field, there are significant deviations between the calculated trajectory with the forward map and the actual trajectory for the particles with large angle or with large momentum difference relative to the particle at the central trajectory. This problem can be solved by using multiple maps for various incident parameters. However, to trace back the particle from the detector after the magnet to the target position, this procedure did not allow COSY to create an inverse map. We employed the novel technique of a neural network [15]. As the position data of the particle at the target position is also known from the beam tracking CRDCs event by event, we have enough information to deduce the rigidity and direction of the particle at the target. A Monte Carlo simulation using the forward map provides a set of events, which is used to train the neural network. Obtained parameters were further tested with another set of simulated events. These steps were repeated until a satisfactory conversion was obtained.

\section{Results and discussion}

Using this technique, the momentum and the velocity vector of the ${ }^{7} \mathrm{Li}$ particles were successfully deduced. The results for angular distributions are displayed in fig. 2. The upper panel shows the net yields for the lead target and the carbon target, respectively, normalized by the number of incoming particles and the target thickness. The contribution from reactions in the thin scintillator in front of the target was subtracted using "blank target" runs. The carbon yield is multiplied by a factor of 6.7 for comparison purpose. The angle of the center of mass velocity of the $n-{ }^{7} \mathrm{Li}$ system relative to the initial ${ }^{8} \mathrm{Li}$ particle is displayed in the center of mass system of ${ }^{8} \mathrm{Li}+$ target. Although the angular dependent acceptance effect is not corrected yet,
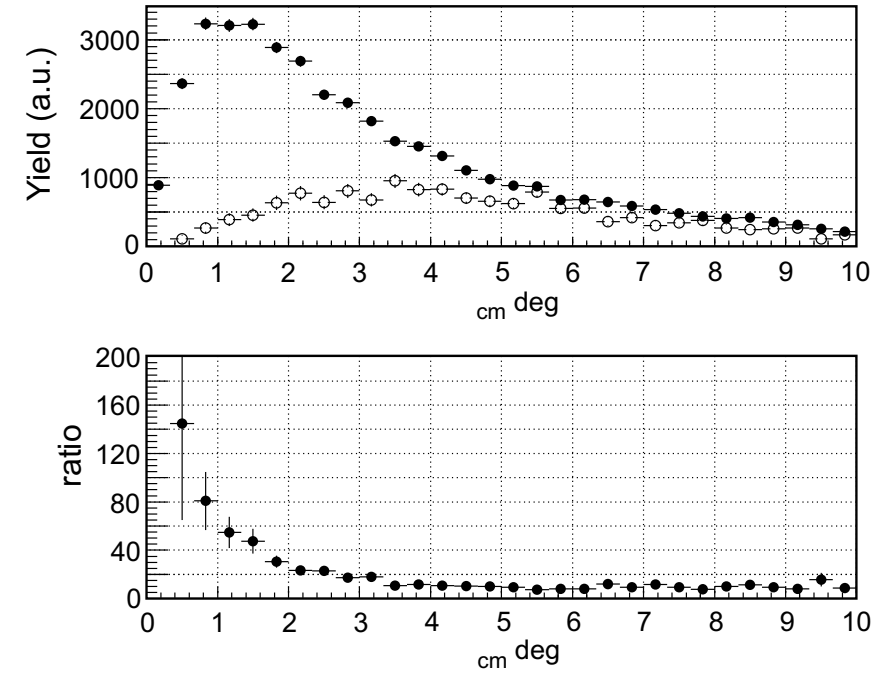

Fig. 2. Angular distribution of $n-{ }^{7} \mathrm{Li}$ coincidence at $41 \mathrm{MeV} / A$. Upper panel: solid symbols are for the lead target and the open circles are for the carbon target. Carbon data is multiplied by factor 6.7 (see text). Lower panel: $\mathrm{Pb} / \mathrm{C}$ ratio of the angular distributions.

a clear difference of the angular distributions for the two targets can be seen. For the lead target, there is a clear enhancement at forward angles less than 6 degrees. This shows the dominance of the Coulomb dissociation process at forward angles. Small yields below 1 degree may correspond to the cutoff of virtual photons i.e., the number of virtual photons whose energy is high enough to dissociate ${ }^{8} \mathrm{Li}(2.033 \mathrm{MeV})$ decreases significantly at the large impact parameter. The carbon target data, on the other hand, shows a rather flat distribution with a maximum at around 3-4 degree. Above 7 degree, which is the grazing angle of the ${ }^{8} \mathrm{Li}+\mathrm{Pb}$ system at $41 \mathrm{MeV} / \mathrm{A}$, the two distributions have the same shape, indicating the nuclear dissociation dominates in these angular regions for both targets. Using the factor 6.7 mentioned above, the magnitude of the two angular distributions matches above 6 degrees. 
The lower panel of the figure shows the ratio between two distributions. This ratio may give a better insight of the target dependence, as the finite acceptance effect is cancelled out in part. For large angles, the ratio is almost a constant. This constant 6.7 corresponds to $A^{2 / 3}$ scaling of lead over carbon. With the often used $A^{1 / 3}$ factor, the two distributions differ in magnitude. As the angle decreases, the ratio gradually becomes larger. At very forward angle, this ratio exceeds 140 which is close to the $Z^{2}$ ratio of the targets. Further analysis, such as angular correlation of the breakup particles ( $n$ and ${ }^{7} \mathrm{Li}$ ) and incident energy dependence, which is now underway, will give more insight to $E 2$ contribution.

In summary, we showed the angular distributions of the Coulomb dissociation for $\mathrm{C}$ and $\mathrm{Pb}$ target at $41 \mathrm{MeV} / A^{8} \mathrm{Li}$ beam energy. Above the grazing angle of the reaction, the nuclear dissociation becomes dominant. The Coulomb dissociation process dominates over nuclear dissociation at forward angles, although nuclear contribution is not negligible below 4 degrees. The present data suggests that a more careful investigation of the angle and energy dependence of the Coulomb dissociation reaction method is clearly necessary to deduce the inverse $(n, \gamma)$ reaction cross section, which is important for the astrophysical nuclear process.

The authors would like to thank to Karlheinz Langanke for emphasizing the importance of the question. Support of the National Science Foundation under grant No. PHY01-10253,
PHY-0354920 and of the OTKA under grant Nos. T42733, T38404, T043585, T049837 are gratefully acknowledged.

\section{References}

1. A.M. Mukhamedzhanov et al., Nucl. Phys. A 725, 279 (2003).

2. G. Baur et al., Prog. Part. Nucl. Phys. 51, 487 (2003).

3. Y. Nagai et al., Phys. Rev. C 71, 055803 (2005).

4. Y. Nagai et al., Astrophys. J. 381, 444 (1991); M. Heil et al., Astrophys. J. 507, 997 (1998).

5. G. Baur, C.A. Bertulani, Nucl. Phys. A 458, 188 (1986).

6. N. Fukuda et al., Phys. Rev. C 70, 054606 (2004).

7. S. Typel et al., Nucl. Phys. A 613, 147 (1997).

8. B. Davids et al., Phys. Rev. Lett. 81, 209 (1998).

9. T. Motobayashi et al., Phys. Rev. Lett. 73, 2680 (1994); F. Schümann et al., Phys. Rev. Lett. 90, 232501 (2003).

10. J. Yurkon et al., Nucl. Instrum. Methods A 422, 291 (1999).

11. T. Baumann et al., Nucl. Instrum. Methods A 543, 517 (2005).

12. B. Luther et al., Nucl. Instrum. Methods A 505, 33 (2003).

13. M.D. Bird et al., IEEE Trans. Appl. Supercond. 15, 1252 (2005).

14. K. Makino, M. Berz, Nucl. Instrum. Methods A 427, 338 (1999).

15. E.g., R. Brun et al., ROOT Users Guide version 4, Chapt. 5 (2005). 